from stimuli in different depth planes, as they would have conjugate points at different retinal disparities.

In any event, Gaze, Keating, Székely and Beazley (Proc. Roy. Soc. B, 175, $107 ; 1970)$ performed experiments the results of which seemed to favour the "post-metamorphosis" hypothesis. They found that the contralateral projection from an eye that had been rotated at an early stage in development was also rotated, but that the ipsilateral projection went to the correct part of the ipsilateral tectum. Furthermore, the ipsilateral projection from the untouched eye was rotated, whereas the contralateral projection was normal. From these results Gaze et al. argued that the ipsilateral fibres could only have grown, under conditions of light stimulation, between neurones that were simultaneously activated by input from each retina. Their experiments were on Xenopus, the clawed toad, once a central figure in pregnancy tests. Jacobson has now repeated these experiments, using a frog, and has added some of the missing controls (Proc. US Nat. Acad. Sci., 68, $528 ; 1971)$.

Jacobson found that animals kept in the dark from larval stage XVII, through metamorphosis, developed normal ipsilateral projections. Indeed, he could detect, by electrophysiological mapping techniques, ipsilateral responses as early as larvae stage XIX, in normal larvae. These results are not consistent with Gaze et al.'s theory, nor is the further result that occlusion of one eye with a skin graft from an early larval stage does not prevent the development of a normal ipsilateral projection. If the skin graft is retained for more than about 120 days, however, the ipsilateral projection becomes disorganized, implying that simultaneous excitation by way of the stimulation of corresponding points on the two retinas may be necessary for the maintenance of binocular function. Jacobson also found that the projections from rotated eyes were rotated, so that there was always an angular disparity between the ipsilateral projection of a rotated eye and the contralateral projection of its unoperated neighbours.

Assuming that Jacobson's and Gaze et al.'s results are comparable in spite of the difference in species used, it seems that there is clear evidence against the view that binocular connexions are determined by visual stimulation, or "experience". They must therefore be genetically determined. The mapping techniques used by both sets of workers are quite crude, however, so it is possible that coarse binocular connexions are made during metamorphosis, but that these become more precise as a result of visual experience. Certainly, binocular excitation is required for the maintenance of these connexions.

\title{
Radio Waves guided by the Earth's Surface
}

Electromagnetic waves are often guided in the laboratory by electromagnetic plumbing in the form of waveguides but it would seem that it is also possible to use the Earth's surface in a similar manner. In next Monday's Nature Physical Science, an extensive study of the propagation of Earth surface waves, including the design of launchers for such waves, is described by A. F. Wickersham of the Stanford Research Institute, California. He points out the possible commercial advantages of radio transmission by surface waves (absence of ionospheric fading and small interference between neighbouring stations, for example) and predicts, perhaps a little overenthusiastically, that a range of 3,700 miles over seawater at $2 \mathrm{MHz}$ might be a possibility.

This is not the first study of Earth surface waves to be undertaken but all the others have relied on the generation of a small amount of energy in the form of transverse magnetic waves by conventional antennas most of whose output is in the transverse electric and magnetic (TEM) mode. Wickersham has used two sizes of tubular launcher to investigate TM surface waves at 115 and $252 \mathrm{MHz}$. He has also employed long aluminium sheets about $30 \mathrm{~cm}$ wide in a successful effort to enhance the quantity of energy received at a distance from the launchers by detectors of flat or tubular design.

One of the principal methods of examining surface wave properties is to measure the falloff of received power with distance from the launcher. For a particular form of TM surface wave known as the Zenneck wave an exponential behaviour would be expected, but an inverse cubic dependence on distance is also possible for other types. The results at $115 \mathrm{MHz}$ over a clay field seem to indicate exponential behaviour but at $252 \mathrm{MHz}$ the cubic law seems to fit the experimental data better. At $252 \mathrm{MHz}$ over salt water and wet clay there is again evidence for a cubic dependence, and because the measurements at this frequency extend to ranges of about $10^{4} \mathrm{~m}$ (at which distance the curvature of the Earth is significant) this is ample evidence of surface wave propagation. If all the data are assessed together, the picture that emerges is of exponential propagation at short distances, cubic propagation at large distances and anomalous propagation at intermediate distances (such as an increasing received power with increasing range).

Wickersham also carried out some experiments with more conventional antennas at $6.91 \mathrm{MHz}$ and found that the radiation component at the surface resembled a surface wave but obeyed a $1 / R^{4}$ law which is in close agreement with a flat Earth rather than a curved Earth theory. No doubt future work on the propagation mechanisms will satisfactorily explain this strange finding.

\section{The Age of Ethiopian Flood Basulis}

IN spite of the importance of the region, so little is known about the geology and geophysics of the African Rift system that any new data are very welcome indeed. But although it is nice to see an oasis after extensive travel in the desert it is even better to come upon a lush river valley. The two potassium-argon dates reported by Rex et al. in next Monday's Nature Physical Science are thus important; but it would have been even more useful to see ten times that number. In the early days of potassium-argon dating, when wonder at the technique was still rife, it was common to see papers reporting but one or two dates. But in this age of the mega-collection for palaeomagnetic and other purposes, it is a little surprising that so few dates are considered sufficient unless, of course, the rest of the collection failed to meet the stringent criteria necessary to ensure success in the use of the particular technique.

This is not, however, to detract from the quality of the two ages that Rex et al. report, nor to underemphasize their intrinsic importance. The dates came from two ignimbrite layers from among the vast flood basalts associated with the Ethiopian Rift and in a limited way put the whole system into some sort of age perspective especially in relation to the even more limited data previously available. The Trap Series of central Ethiopia, for example, is often regarded as Eocene-Oligocene, a range which receives some support from a $49 \pm 15$ million year radiometric age from the Blue Nile gorge. $A$ palaeomagnetic study of rocks in the Addis Ababa area, on the other hand, has yielded a pole position more consistent with the Miocene. This highlights well the problems inherent in too few data. It would, in turn, be dangerous to conclude too much from the new dates of 9 and 21 million years. What they do suggest, however, is a long period of basalt extrusion in the Ethiopian Rift system. 